Article

\title{
Assessments of Facial Muscle Thickness by Ultrasound in Younger Adults: Absolute and Relative Reliability
}

\author{
Takashi Abe ${ }^{1, *}$, Robert W. Spitz ${ }^{1}$, Vickie Wong ${ }^{1}$, Ricardo B. Viana ${ }^{1,2}{ }^{\circledR}$, Yujiro Yamada ${ }^{1}$, \\ Zachary W. Bell ${ }^{1}$, Raksha N. Chatakondi ${ }^{1}$ and Jeremy P. Loenneke ${ }^{1}$ \\ 1 Department of Health, Exercise Science, \& Recreation Management, Kevser Ermin Applied Physiology \\ Laboratory, The University of Mississippi, University, MS 38677, USA; rwspitz@go.olemiss.edu (R.W.S.); \\ vwong@go.olemiss.edu (V.W.); vianaricardoborges@hotmail.com (R.B.V.); yyamada1@go.olemiss.edu (Y.Y.); \\ zwbell@go.olemiss.edu (Z.W.B.); rnchatak@go.olemiss.edu (R.N.C.); jploenne@olemiss.edu (J.P.L.) \\ 2 Faculty of Physical Education and Dance, Federal University of Goiás, Goiânia 74690-900, Brazil \\ * Correspondence: t12abe@gmail.com; Tel.: +1-(662)-915-5521; Fax: +1-(662)-915-5525
}

Received: 8 October 2019; Accepted: 6 November 2019; Published: 7 November 2019

\begin{abstract}
The absolute reliability (i.e., standard error of measurement and minimal difference) of a measurement is important to consider when assessing training effects. However, the absolute reliability for ultrasound measured facial muscle thickness had not been investigated. In order to examine the absolute and relative reliability of measuring facial muscles, 98 healthy, young, and middle-aged adults (18-40 years) had ultrasound measurements taken twice, separated by an average of three days. Six facial muscles were selected to determine the reliability of facial muscle thickness. The relative reliability ( $\mathrm{ICC}_{3,1}$ ) ranged from 0.425 for the orbicularis oris (inferior) to 0.943 for the frontalis muscle. The absolute reliability (minimal difference) ranged from $0.25 \mathrm{~mm}$ for the orbicularis oculi to $1.82 \mathrm{~mm}$ for the masseter. The percentage minimal difference was $22 \%, 25 \%, 26 \%, 29 \%, 21 \%$, and $10 \%$ for the frontalis, orbicularis oculi, orbicularis oris (superior), orbicularis oris (inferior), depressor anguli oris, and masseter, respectively. Our results indicated that the relative reliability was similar to that observed previously. The absolute reliability indicated that the measurement error associated with measuring muscle thickness of the face may be greater than that of the trunk/limb muscles. This may be related to the difficulty of accurately determining the borders of each muscle.
\end{abstract}

Keywords: reproducibility; facial muscle thickness; ultrasonography

\section{Introduction}

As humans age, it is well-known that wrinkles and sagging can appear on the face. For most people, facial wrinkles tend to appear in similar areas of the face (i.e., forehead, eye, mouth, cheek) [1]. The curvatures of wrinkles are often similar in these locations. The literature suggests that the repeated occurrence as well as transition between differing facial expressions results in a groove forming beneath the skin surface, after which fine wrinkles develop [1,2]. To lessen the development of facial wrinkles, several studies have observed that short-term facial muscle exercise training improves wrinkles and prevents sagging of the face in middle-aged and older women [3-5]. This may also suggest that the influence on facial soft tissue is different between facial exercises and movements of differing facial expressions. If our facial muscles are primarily influenced by facial exercise/movements, it is necessary to accurately evaluate facial muscle integrity in order to determine age-related changes and the effects of exercise on facial muscle size and function [6].

Brightness-mode (B-mode) ultrasound is a tool that can be used for assessing facial muscle size (i.e., muscle thickness and muscle cross-sectional area) in humans. Several studies have 
investigated the reliability of facial muscle thickness measurements when using ultrasound [7-11]. However, these studies only reported intra-rater correlation coefficients (ICC). Compared to the results of extremity and trunk muscles [12,13], the ICC values of facial muscles were relatively low. ICC is a relative measure of reliability [14], but a measure of the absolute reliability (i.e., standard error of measurement and minimal difference) of facial muscle thickness by ultrasound has not been previously investigated [6]. In order to compare differences between groups, as well as the possibility of any training effect, the reliability reported in absolute values should yield further transparency when interpreting the data. Thus, the purpose of this study was to examine the test-retest reliability of ultrasound-measured facial muscle thickness in a large sample of young and middle-aged adults.

\section{Methods}

\subsection{Participants}

A total of 98 healthy young and middle-aged adults (men $=46$, women $=52)$, between the ages of 18 and 40, were measured twice (Visit- 1 and Visit-2) with a minimum interval of $24 \mathrm{~h}$ and a maximum of six days between visits (average, three days). Participants reported no history of head or facial trauma, head trauma, facial palsy, or any other neurological disorder. All participants signed a written informed consent to participate in the study. This study was approved by the University of Mississippi Institutional Review Board $(\# 19-107,2019)$. Participants were instructed to refrain from any vigorous physical activity, as well as to not consume alcohol, for $24 \mathrm{~h}$ prior to testing sessions.

\subsection{Measurements of Facial Muscle Thickness}

Participants were measured in a supine position, with their head supported by a small pillow and the investigator seated behind their head. The same investigator completed all ultrasound measurements for this study (i.e., intra-observer reliability). Participants were instructed to relax their facial muscles during measurements. Facial muscle thickness was measured using B-mode ultrasound with a 13-MHz linear transducer (Logiq e; GE, Fairfield, CT, USA) on the right side of the face only. The electrical caliper built into the device can measure muscle thickness in 0.1-mm increments. During the pilot stage, the researchers completed imaging of 10 different facial muscles, as referenced by previous research [7-10]. Sites were selected that allowed for highly reproducible images and the maintenance of a reliable probe positioning. In addition, it was confirmed that these muscles were contoured from surrounding bone and collagen, with appropriate connective tissue fascia beneath them ensuring a reproducible and consistent aim of the ultrasound beam. For the current study, six muscles were selected (frontalis, orbicularis oculi, orbicularis oris pars marginalis superior, orbicularis oris pars marginalis inferior, depressor anguli oris, and masseter) to determine the reliability of facial muscle thickness (Figure 1). Two separate images were obtained at each site and stored for offline analysis following data collection. Analyses of ultrasound images were performed randomly after all measurements were completed. Furthermore, analyses were performed for the second measurement following the first measurement (i.e., all of the Visit 1 measurements were randomly analyzed prior to analyzing the Visit 2 measurements). To determine muscle thickness, muscle boundaries were manually determined by an experienced ultrasonographer. Muscle thickness was quantified as the maximum transverse diameter of each muscle. Body mass and standing height were measured to the nearest $0.1 \mathrm{~kg}$ and $0.1 \mathrm{~cm}$, respectively, using an electronic weight scale and a portable stadiometer (both Seca 220; Hamburg, Germany) following paperwork and prior to any testing. 


a) Frontalis
b) Orbicularis oculi
c) Orbicularis oris (superior)
d) Orbicularis oris (inferior)
e) Depressor anguli oris
f) Masseter

Figure 1. Ultrasound measurements of the frontalis (a), orbicularis oculi (b), orbicularis oris pars marginalis superior (c), orbicularis oris pars marginalis inferior (d), depressor anguli oris (e), and masseter (f) muscles. Measurement sites were selected that allowed for highly reproducible images and the maintenance of a reliable probe positioning, as referenced by previous studies [7-11]. Muscle thickness is indicated in the yellow bar for each muscle.

\subsection{Statistical Analysis}

Data are presented as mean and standard deviation (SD). The mean and SD of the difference between Visit-1 and Visit-2 was calculated for facial muscle thickness measured at six sites. The minimal difference (MD) was formulated as follows [14]:

$$
\begin{gathered}
\mathrm{SEM}=\mathrm{SDd} / \sqrt{2} \\
\mathrm{MD}=\mathrm{SEM} \times 1.96 \times \sqrt{2}=\mathrm{SDd} \times 1.96
\end{gathered}
$$


where SEM is the standard error of measurement and SDd is the SD of the difference between the testing visits.

The relative minimal difference was calculated by taking the minimal difference and then dividing by the grand mean. Intraclass-correlations $\left(\mathrm{ICC}_{3,1}\right)$ were performed to determine the associations between Visit-1 and Visit-2. ICC 3,1 is commonly used in the literature and is implemented to illustrate the reliability within a given laboratory. The percentage coefficient of variation was calculated as the SD between the two measurements divided by the mean of the two measurements. All data analyses were performed using IBM SPSS Statistics version 25.

\section{Results}

Mean age, standing height, body mass, and body mass index of the participants ( $\mathrm{n}=98)$ were 23.9 (4.8) years, $169.3(9.6) \mathrm{cm}, 69.3(14.9) \mathrm{kg}$, and $23.9(3.6) \mathrm{kg} / \mathrm{m}^{2}$, respectively. Approximately half of the participants $(n=47)$ reported performing weight-lifting exercise on their major muscle groups for a minimum of two years.

The relative reliability $\left(\mathrm{ICC}_{3,1}\right)$ ranged from 0.425 for the orbicularis oris (inferior) to 0.943 for the frontalis muscle. The absolute reliability (minimal difference) ranged from $0.25 \mathrm{~mm}$ for the orbicularis oculi to $1.82 \mathrm{~mm}$ for the masseter. The percentage coefficient of variation was the lowest for the masseter at $2.8 \%$ and greatest for the orbicularis oris (inferior) at $8.2 \%$ (Table 1 ). The percentage minimal difference was $22 \%, 25 \%, 26 \%, 29 \%, 21 \%$, and $10 \%$ for the frontalis, orbicularis oculi, orbicularis oris (superior), orbicularis oris (inferior), depressor anguli oris, and masseter, respectively.

Table 1. Test-retest reliability of ultrasound measured facial muscle thickness in younger adults $(\mathrm{n}=98)$. All muscle thickness values are presented in millimeters.

\begin{tabular}{ccccccc}
\hline Muscle & Visit 1 (SD) & Visit 2 (SD) & Difference (SD) & Minimal Difference & \%CV & ICC $_{\mathbf{3}, \mathbf{1}}$ \\
\hline Frontalis & $1.98(0.69)$ & $1.98(0.66)$ & $0.00(0.23)$ & 0.45 & 6.2 & 0.943 \\
Orbicularis oculi & $0.96(0.31)$ & $0.98(0.30)$ & $-0.02(0.13)$ & 0.25 & 7.7 & 0.904 \\
Orbicularis oris (sup) & $1.53(0.27)$ & $1.55(0.24)$ & $-0.02(0.21)$ & 0.41 & 7.6 & 0.646 \\
Orbicularis oris (inf) & $1.41(0.20)$ & $1.48(0.21)$ & $-0.06(0.22)$ & 0.43 & 8.2 & 0.425 \\
Depressor anguli oris & $3.36(0.77)$ & $3.33(0.75)$ & $0.03(0.37)$ & 0.73 & 5.7 & 0.880 \\
Masseter & $17.0(2.7)$ & $17.0(2.6)$ & $0.02(0.93)$ & 1.82 & 2.8 & 0.938 \\
\hline
\end{tabular}

Data are presented as mean and standard deviation (SD). $\% \mathrm{CV}$, percentage coefficient of variation; ICC, intraclass correlation coefficient; Orbicularis oris (sup), orbicularis oris pars marginalis superior; Orbicularis oris (inf), orbicularis oris pars marginalis inferior.

\section{Discussion}

In this study, we calculated not only the relative reliability $\left(\mathrm{ICC}_{3,1}\right)$ but also the absolute reliability (minimal difference) of six separate facial muscles using B-mode ultrasound. Our $\mathrm{ICC}_{3,1}$ values were similar to the values reported in previous studies [7-9], with large differences depending on the facial muscle measured (ranging between 0.33 and 0.93). Compared to measuring muscle thickness of the limb and trunk muscles, there are notable challenges to measuring the facial muscles. For example, when measuring the limbs and trunk, there is a relatively clear boundary (i.e., fascia connective tissue) between the subcutaneous adipose tissue and muscle or between each muscle on the ultrasound image. In the facial muscles, however, the boundaries between the adipose tissue and muscle can be difficult to determine. In other words, making a mistake in determining the boundary can result in a large degree of measurement error. In addition to the relative reliability, the reliability reported in absolute values is also important when interpreting the data in order to compare differences between groups and assess possible training effects.

To the best of our knowledge, the current study is the first to provide information about the absolute reliability of facial muscle thickness measurements by ultrasound. The absolute minimal difference was less than $1.0 \mathrm{~mm}$ in the facial muscles, except for the masseter. However, the size of facial muscles were relatively small compared to the limb/trunk muscles. In the present study, 
the relative minimal difference values were over $20 \%$ of the mean values for five of the six muscles. When investigating the effect of exercise on facial muscle size, one study demonstrated changes across time relative to a time-matched control group [15]. However, not all studies included this control group. For example, one study measured facial muscle thickness using ultrasound before and after eight weeks of facial muscle training [3] and found a pre-post difference in muscle thickness of the zygomatic major, levator labii superioris, orbicularis, and digastric muscle in the training group. The percentage increase in muscle thickness of these muscles was approximately $\sim 8 \%$ for all muscles measured. This study, however, did not have a control group and did not provide the reliability of their measurements. If we compare their change in orbicularis oris size with our reliability, the change would not be different from measurement error. Although we cannot rule out the possibility that they observed a change, we must consider the possibility that the results would not exceed that of the measurement error. It should also be noted that many studies assess long-term (i.e., months) changes in variables but rely on a short test-retest (i.e., a few days) periods for the error (i.e., minimal difference) needed to determine meaningful changes [16-18]. The results of this study were determined by short-term (average, three days) assessment of reliability. If long-term reproducibility is determined, the minimal difference may become even larger [19].

For improved accuracy with future measurements, methods to reduce measurement error when assessing facial muscles should be considered. When assessing limb muscles, the measurement position is determined based on anatomical landmarks (e.g., percentage of limb length). The orbicularis oculi and orbicularis oris surround the eyes and mouth, respectively, making it easy to predict the position of the muscle. However, there is difficultly when identifying the origin and the insertion of each muscle in many of the other facial muscles. Although some studies [7-9] have explained the position of the measurement site with a picture, not all participants have the target muscle in the same position. It may be important to improve the reproducibility of the probe position using the landmark of the face, but that alone is unlikely to solve all issues. Therefore, further solutions are needed. It is possible that real-time ultrasound can display isolated muscle activity of the facial muscles during movement of facial expression such as elevating eyebrows, closing eyelids, and chewing teeth. If the position and boundary are not clear, the above-described inspection using the muscle movement before measurement may be something to consider in order to ensure proper position [20]. Furthermore, our muscle thickness measurements were performed in units of $0.1 \mathrm{~mm}$, but studies using different ultrasound devices may have different levels of detection. The impact that this has on the reliability should also be considered.

In conclusion, our study provided the relative and absolute reliability for assessing muscle thickness of the face. Our results indicated that the relative reliability was similar to that observed previously, but the absolute reliability had not been previously reported. The measurement error associated with measuring muscle thickness of the face may be greater than that of the trunk/limb muscles. This is due to the difficulty of accurately determining the borders of each muscle. Taken together, these findings highlight the importance of including time-matched control groups in order to capture not only tester error, but also errors related to random biological fluctuations within a specific time frame.

Author Contributions: T.A., R.W.S., V.W., R.B.V. and J.P.L. conceived and designed the study. T.A., R.W.S., V.W., R.B.V., Y.Y. and R.N.C. performed the data collection. T.A. and J.P.L. analyzed the data. T.A. wrote the manuscript. Z.W.B., R.W.S., V.W., R.B.V., Y.Y., R.N.C. and J.P.L. reviewed and critically revised the manuscript. All authors approved the final version of the manuscript.

Funding: The authors received no financial support for the research, authorship, and publication of this article.

Acknowledgments: The authors would like to thank the individuals who voluntarily gave their time to participate in this study.

Conflicts of Interest: The authors declared no conflicts of Interests with respect to the research, authorship, and publication of this article. 


\section{References}

1. Lemperle, G.; Holmes, R.E.; Cohen, S.R.; Lemperle, S.M. A classification of facial wrinkles. Plast. Reconstr. Surg. 2001, 108, 1735-1750. [CrossRef] [PubMed]

2. Calleja-Agius, J.; Brincat, M.; Borg, M. Skin connective tissue and ageing. Best Pract. Res. Clin. Obstet. Gynaecol. 2013, 27, 727-740. [CrossRef] [PubMed]

3. Hwang, U.; Kwon, O.; Jung, S.; Ahn, S.; Gwak, G. Effect of a facial muscle exercise device on facial rejuvenation. Aesthet. Surg. J. 2018, 38, 463-476. [CrossRef] [PubMed]

4. Van Borsel, J.; De Vos, M.-C.; Bastiaansen, K.; Welvaert, J.; Lambert, J. The effectiveness of facial exercise for facial rejuvenation: A systematic review. Aesthet. Surg. J. 2014, 34, 22-27. [CrossRef] [PubMed]

5. Alam, M.; Walter, A.J.; Geisler, A.; Roongpisuthipong, W.; Sikorski, G.; Tung, R.; Poon, E. Association of facial exercise with the appearance of aging. JAMA Dermatol. 2018, 154, 365-367. [CrossRef] [PubMed]

6. Abe, T.; Loenneke, J.P. The influence of facial muscle training on the facial soft tissue profile: A brief review. Cosmetics 2019, 6, 50. [CrossRef]

7. Satiroglu, F.; Arun, T.; Isik, F. Comparative data on facial morphology and muscle thickness using ultrasonography. Eur. J. Orthodont. 2005, 27, 562-567. [CrossRef] [PubMed]

8. Alfen, N.V.; Gilhuis, H.J.; Keijzers, J.P.; Pillen, S.; Van Dijk, J.P. Quantitative facial muscle ultrasound: Feasibility and reproducibility. Muscle Nerve 2013, 48, 375-380. [CrossRef] [PubMed]

9. Volk, G.F.; Wystub, N.; Pohlmann, M.; Finkensieper, M.; Chalmers, H.J.; Guntinas-Lichius, O. Quantitative ultrasonography of facial muscles. Muscle Nerve 2013, 47, 878-883. [CrossRef] [PubMed]

10. Volk, G.F.; Sauer, M.; Pohlmann, M.; Guntinas-Lichius, O. Reference values for dynamic facial muscle ultrasonography in adults. Muscle Nerve 2014, 50, 348-357. [CrossRef] [PubMed]

11. Emshoff, R.; Bertram, S.; Strobl, H. Ultrasonographic cross-sectional characteristics of muscles of the head and neck. Oral Surg. Oral Med. Oral Pathol. Oral Radiol. Endod. 1999, 87, 93-106. [CrossRef]

12. Abe, T.; Loenneke, J.P.; Thiebaud, R.S.; Loftin, M. Morphological and functional relationships with ultrasound measured muscle thickness of the upper extremity and trunk. Ultrasound 2014, 22, 229-235. [CrossRef] [PubMed]

13. Abe, T.; Loenneke, J.P.; Thiebaud, R.S. Morphological and functional relationships with ultrasound measured muscle thickness of the lower extremity: A brief review. Ultrasound 2015, 23, 166-173. [CrossRef] [PubMed]

14. Weir, J.P. Quantifying test-retest reliability using the intraclass correlation coefficient and the SEM. J. Strength Cond. Res. 2005, 19, 231-240. [PubMed]

15. Kavanagh, S.; Newell, J.; Hennessy, M.; Sadick, N. Use of a neuromuscular electrical stimulation device for facial muscle toning: A randomized, controlled trial. J. Cosmetic Dermatol. 2012, 11, 261-266. [CrossRef] [PubMed]

16. Loenneke, J.P.; Rossow, L.M.; Fahs, C.A.; Thiebaud, R.S.; Mouser, G.J.; Bemben, M.G. Time-course of muscle growth, and its relationship with muscle strength in both young and older women. Geriatr. Gerontol. Int. 2017, 17, 2000-2007. [CrossRef] [PubMed]

17. Ogasawara, R.; Yasuda, T.; Ishii, N.; Abe, T. Comparison of muscle hypertrophy following 6-month of continuous and periodic strength training. Eur. J. Appl. Physiol. 2013, 113, 975-985. [CrossRef] [PubMed]

18. Thiebaud, R.S.; Loenneke, J.P.; Fahs, C.A.; Rossow, L.M.; Kim, D.; Abe, T.; Anderson, M.A.; Young, K.C.; Bemben, D.A.; Bemben, M.G. The effects of elastic band resistance training combined with blood flow restriction on strength, total bone-free lean body mass and muscle thickness in postmenopausal women. Clin. Physiol. Func. Imaging 2013, 33, 344-352. [CrossRef] [PubMed]

19. Abe, T.; Dankel, S.J.; Buckner, S.L.; Jessee, M.B.; Mattocks, K.T.; Mouser, J.G.; Bell, Z.W.; Loenneke, J.P. Short term (24 hours) and long term (1 year) assessments of reliability in older adults: Can one replace the other? J. Aging Res. Clin. Pract. 2018, 7, 82-84.

20. Abe, T.; Loenneke, J.P. Author's response. Assessing forearm muscle size with the ultrasound. Clin. Physiol. Func. Imaging 2018, 38, 1069-1070. [CrossRef] [PubMed]

(C) 2019 by the authors. Licensee MDPI, Basel, Switzerland. This article is an open access article distributed under the terms and conditions of the Creative Commons Attribution (CC BY) license (http://creativecommons.org/licenses/by/4.0/). 